\title{
On the spectrum and spacetime supersymmetry of heterotic ambitwistor string
}

\author{
Henrique Flores and Matheus Lize \\ ICTP South American Institute for Fundamental Research, \\ Instituto de Física Teórica, Universidade Estadual Paulista, \\ Rua Dr. Bento Teobaldo Ferraz 271, São Paulo, SP Brasil \\ E-mail: henrique.flores@unesp.br, matheus.lize@unesp.br
}

ABSTRACT: We analyse the BRST cohomology of the Ramond sector of heterotic ambitwistor theory. We also write the free string field theory action and compute the supersymmetry transformations.

KEYwords: BRST Quantization, String Field Theory, Superstrings and Heterotic Strings ARXIV EPRINT: 1903.05792 


\section{Contents}

1 Introduction 1

2 Ambitwistor action and Ramond sector 2

3 Cohomology 3

3.1 Vertex operators 4

3.2 Equations of motion and gauge symmetries 4

3.3 Gauge-fixing and independent equations of motion 6

4 Action $\quad 8$

5 Supersymmetry $\quad 9$

$\begin{array}{lll}5.1 & \text { Supersymmetry transformations of NS and R sectors } & 10\end{array}$

$\begin{array}{ll}5.2 \text { Supersymmetry invariance of the action } & 11\end{array}$

5.3 Supersymmetry for $\left(h_{m n}, t, \mathbf{i}, \mathbf{d}\right) \quad 12$

5.4 Supersymmetry for $\left(H_{m n p}, C_{m n p}, \mathbf{d}_{m}^{\alpha}, \mathbf{i}_{\alpha}\right) \quad 13$

A Ramond sector, cocycles and Gamma matrices $\quad 14$

$\begin{array}{lll}\text { A.1 The Ramond sector } & 14\end{array}$

$\begin{array}{ll}\text { A.2 Bosonization and cocycles } & 14\end{array}$

\section{Introduction}

The ambitwistor string was introduced by Mason and Skinner in [1] as a string theory whose tree amplitudes reproduce the Cachazo-He-Yuan formulas for massless scattering [2]. It contains only left-moving worldsheet fields and has no massive states. As described in [1], it is the $\alpha^{\prime} \rightarrow 0$ limit of superstring theory, even though only the $G S O(+)$ sector of the Type II version correctly describes $d=10 N=2$ supergravity.

The spectra was identified in [3] using the standard BRST method where equations of motion and gauge invariances are derived from the cohomology at ghost-number 2 of the BRST operator. The quadratic term for the string field theory action was also constructed in a gauge-invariant manner; and except for the $G S O(+)$ sector of the Type II ambitwistor string, the kinetic terms for the other ambitwistor strings are non-unitary, i.e. they contain more than two derivatives. In this paper, we focus on the Ramond sector of heterotic ambitwistor string. We want to understand if the fermionic spectra of the theory is given by non-unitary equations of motion, and study the supersymmetry transformations of these non-unitary systems.

Section 2 reviews the ambitwistor model and sets our notation. It can be skipped on a first reading. 
We start in section 3, where we use the standard BRST method to compute the equations of motion of the Ramond sector for the heterotic system. These represent the fermionic degrees of freedom of the theory, and our analysis shows that they also follow non-unitary equations of motion. We write a gauge-invariant version of theory in terms of Fronsdal fields [4]. The kinetic term of the fermionic ambitwistor string field theory action is also computed in section 4. It is expressed in terms of gauge-invariant objects and resembles Fronsdal's free action despite having more derivatives.

Finally, in section 5 we write the supersymmetry transformations of the system. In RNS language, the supersymmetry operator is defined on-shell and thus gives the supersymmetry transformations up to equations of motion. Then we prove the invariance of the action under supersymmetry transformations.

\section{Ambitwistor action and Ramond sector}

We first review the ambitwistor model. Its main purpose is to set the basic definitions and notation.

The heterotic ambitwistor model is defined by the free action

$$
S=\frac{1}{2 \pi} \int \mathrm{d}^{2} z\left(\mathrm{p}_{m} \bar{\partial} x^{m}+\psi_{m} \bar{\partial} \psi^{m}+b \bar{\partial} c+\tilde{b} \bar{\partial} \tilde{c}+\beta \bar{\partial} \gamma+S_{\mathrm{j}}\right)
$$

where $\mathrm{p}_{m}$ is a worldsheet holomorphic one-form and $x^{m}$ is an holomorphic coordinate function. The $b$ and $c$ fields together with $\beta$ and $\gamma$ are the Faddeev-Popov ghosts of superconformal worldsheet symmetry. Particular to the heterotic model, we have the current action $S_{\mathrm{j}}$; its specific form is irrelevant for us, we only require the existence of a current $\mathrm{j}^{a}$ with conformal weight 1 that satisfies the OPE

$$
\mathrm{j}^{a}(z) \mathrm{j}^{b}(w) \sim \frac{\delta^{a b}}{(z-w)^{2}}+\frac{f_{c}^{a b} \mathrm{j}^{c}(w)}{(z-w)}
$$

being $f_{c}^{a b}$ the structure constants of the Lie algebra in question. The Ambitwistor model differs from the superstring due to the presence of the $\tilde{b}$ and $\tilde{c}$ ghosts related to the gauge symmetries of the light-cone constrain: $\mathrm{p}^{2}=0$. These ghosts have conformal weights 2 and -1 respectively and both are worldsheet fermions.

Our Majorana spinors $\psi^{m}$ will be rewritten in the complex linear combinations:

$$
\psi^{ \pm i}=\frac{1}{\sqrt{2}}\left(\psi^{2 i-1} \mp i \psi^{2 i}\right)
$$

for $i=1, \ldots, 5$ that are subsequently bosonized to

$$
\psi^{ \pm i}(z)=\exp \left( \pm \phi_{i}(z)\right) c_{ \pm e_{i}}
$$

with $\phi$ 's satisfying

$$
\phi_{i}(z) \phi_{j}(\mathrm{w}) \sim+\delta_{i j} \ln (z-\mathrm{w})
$$


The $(\beta, \gamma)$ system is bosonized with extra fermions $(\xi, \eta)[5]$, both primaries of conformal weight 0 and 1 respectively:

$$
\beta=\partial \xi e^{-\phi_{6}} c_{e_{6}} \quad \text { and } \quad \gamma=\eta e^{\phi_{6}} c_{e_{6}} .
$$

This choice follows the conventions of [6] and [7] where we have introduced the cocycles $c_{e_{i}}$ and $c_{e_{6}}$. During the computation of cohomology, cocycle factors are important and must be taken into account. The definition of cocycles depends on the way we order the different $\phi_{i}$. For us the chiral bosons corresponding to $\psi^{m}$ are ordered from 1 to 5 while the boson coming from the $\beta \gamma$ system is labeled as 6 . A review of how to operate with cocycles can be found in [6] and a brief explanation is written in appendix A. The sixth boson has OPE:

$$
\phi_{6}(z) \phi_{6}(\mathrm{w}) \sim-\ln (z-\mathrm{w})
$$

while $(\xi, \eta)$ form a free system:

$$
\xi(z) \eta(\mathrm{w}) \sim \frac{1}{(z-\mathrm{w})}
$$

The symmetries of this action are encoded in the following BRST charge:

$$
Q=\oint \frac{d z}{2 \pi i}\left[c\left(\mathrm{~T}_{\text {matter }}+T_{\tilde{b} \tilde{c}}+T_{\beta \gamma}+\mathrm{T}_{\mathrm{j}}\right)+b c \partial c+\frac{1}{2} \tilde{c} \mathrm{p}^{2}+\gamma \mathrm{p}^{m} \psi_{m}-\gamma^{2} \tilde{b}\right]
$$

provided

$$
\begin{aligned}
\mathrm{T}_{\text {matter }} & =-\mathrm{p}_{m} \partial x^{m}+\frac{1}{2} \sum_{i=1}^{5} \partial \phi_{i} \partial \phi_{i}, & \mathrm{~T}_{\tilde{b} \tilde{c}}=\tilde{c} \partial \tilde{b}-2 \tilde{b} \partial \tilde{c} \\
T_{\beta \gamma}=-\frac{1}{2} \partial \phi_{6} \partial \phi_{6}-\partial^{2} \phi_{6}-\eta \partial \xi, & \text { and } & \gamma^{2}=\eta \partial \eta e^{+2 \phi_{6}}
\end{aligned}
$$

These are all the stress-energy tensors for $\left(x^{m}, \mathrm{p}_{m}, \psi^{m}\right),(\beta, \gamma)$ and $(\tilde{b}, \tilde{c})$. We only require for the stress tensor of the current sector, $\mathrm{T}_{\mathrm{j}}$, that the following OPE is satisfied:

$$
\mathrm{T}_{\mathbf{j}}(z) \mathrm{T}_{\mathbf{j}}(w) \sim \frac{c_{j}}{2(z-w)^{4}}+\frac{2 \mathrm{~T}_{\mathbf{j}}(w)}{(z-w)^{2}}+\frac{\partial \mathrm{T}_{\mathbf{j}}(w)}{(z-w)} .
$$

Then, provided the central charge of the current system is 16 , it is possible to show that $Q^{2}=0$ when the spacetime is 10-dimensional.

\section{Cohomology}

In this section, we compute the ghost number 2 BRST cohomology of the Ambitwistor string for states in the Ramond sector. The cohomology of the Neveu-Schwarz sector has already been computed in [3].

We start by writing the most general vertex operator and the most general gauge parameter. Once all equations of motion and gauge transformations are obtained, we solve the algebraic gauge conditions to obtain a set of independent field equations. 


\subsection{Vertex operators}

States are defined by picture number $-1 / 2$ and ghost number 2 BRST cohomology. We define ghost and picture numbers by the expressions:

$$
N_{\text {ghost }}=-\oint \frac{\mathrm{d} z}{2 \pi i}(b c+\tilde{b} \tilde{c}+\xi \eta) \quad \text { and } \quad N_{\text {picture }}=\oint \frac{\mathrm{d} z}{2 \pi i}\left(\xi \eta-\partial \phi_{6}\right) .
$$

Vertex operator. The most general ghost number 2 and picture number $-1 / 2$ vertex operator that is annihilated by $b_{0}$ is given by the sum,

$$
V_{R}=V_{+}+V_{-},
$$

where $V_{+}$and $V_{-}$are the $G S O(+)$ and $G S O(-)$ combinations. The $G S O(+)$ vertex operator is given by:

$$
\begin{aligned}
V_{+}= & c \eta S^{\alpha} e^{\phi / 2} \mathbf{A}_{\alpha}+\tilde{c} \eta S^{\alpha} e^{\phi / 2} \mathbf{B}_{\alpha}+c \tilde{c} S^{\dot{\alpha}} e^{-\phi / 2} \partial x^{m} \mathbf{C}_{m \dot{\alpha}}+c \tilde{c} S^{\dot{\alpha}} e^{-\phi / 2} \mathrm{p}_{m} \mathbf{D}_{\dot{\alpha}}^{m} \\
& +c \tilde{c} S^{\dot{\alpha}} e^{-\phi / 2} \mathbf{j}^{a} \mathbf{E}_{\dot{\alpha}}^{a}+c \partial \tilde{c} S^{\dot{\alpha}} e^{-\phi / 2} \mathbf{F}_{\dot{\alpha}}+c \tilde{c} S^{\dot{\alpha}} \partial e^{-\phi / 2} \mathbf{G}_{\dot{\alpha}} \\
& +c \tilde{c} \psi_{m}(\psi S)^{\alpha} e^{-\phi / 2} \mathbf{H}_{\alpha}^{m}+c \tilde{c} \partial \tilde{c} \partial \xi S^{\alpha} e^{-3 \phi / 2} \mathbf{I}_{\alpha}+\tilde{c} \partial \tilde{c} S^{\dot{\alpha}} e^{-\phi / 2} \mathbf{J}_{\dot{\alpha}}
\end{aligned}
$$

while $V_{-}$is obtained from $V_{+}$by changing the chirality of our spinors. Notice that the vertices $\psi^{m} \psi^{n} S^{\dot{\alpha}}$ and $\partial S^{\dot{\alpha}}$ have not been written. In bosonized form, these combinations are related to $\psi \psi S$ via field redefinitions [6]; there is no need to worry about them.

Gauge vertex. As for the gauge transformations, we parametrize them by ghost number 1 and picture number $-1 / 2$ vertex operators:

$$
\Lambda=c S^{\dot{\alpha}} e^{-\phi_{6} / 2} \lambda_{\dot{\alpha}}+\tilde{c} S^{\dot{\alpha}} e^{-\phi_{6} / 2} \omega_{\dot{\alpha}}+c \tilde{c} \partial \xi S^{\alpha} e^{-3 \phi_{6} / 2} \mu_{\alpha} .
$$

Both expressions (3.2) and (3.4) constitute the basic field content of BRST cohomology.

\subsection{Equations of motion and gauge symmetries}

For clarity we consider only the $G S O(+)$ sector. The $G S O(-)$ is obtained by replacing chiral indices for anti-chiral and vice-versa. We present the equations of motion organized by ghost number as they were obtained from the OPE of $Q$ and $V_{+}$. We also write the worldsheet operator that multiplies the resulting equation of motion.

- For $(2 c, 1 \tilde{c})$ multiplying $\left(S^{\dot{\alpha}} e^{-\phi_{6} / 2} c \tilde{c} \partial^{2} c\right)$ :

$$
+\frac{1}{2} \partial_{m} \mathbf{D}_{\dot{\alpha}}^{m}+\mathbf{F}_{\dot{\alpha}}-\frac{3}{8} \mathbf{G}_{\dot{\alpha}}-\frac{9}{4}\left(\Gamma^{m}\right)_{\dot{\alpha}}^{\beta} \mathbf{H}_{m \beta}=0
$$

- For $(0 c, 1 \tilde{c})$ multiplying $\left(S^{\dot{\alpha}} e^{3 \phi_{6} / 2} \tilde{c} \eta \partial \eta\right)$ :

$$
+\mathbf{J}_{\dot{\alpha}}-\frac{i}{\sqrt{2}}\left(\Gamma^{m}\right)_{\dot{\alpha}}^{\beta} \partial_{m} \mathbf{B}_{\beta}=0
$$

- For $(1 c, 0 \tilde{c})$ multiplying $\left(S^{\dot{\alpha}} e^{3 \phi_{6} / 2} c \eta \partial \eta\right)$ :

$$
-\frac{i}{\sqrt{2}}\left(\Gamma^{m}\right)_{\dot{\alpha}}^{\beta} \partial_{m} \mathbf{A}_{\beta}-\mathbf{G}_{\dot{\alpha}}+\mathbf{F}_{\dot{\alpha}}=0
$$


- For $(1 c, 2 \tilde{c})$

- multiplying $\left(S^{\alpha} e^{-\phi_{6} / 2} c \tilde{c} \partial \tilde{c} \mathrm{p}_{m}\right)$ :

$$
-\frac{1}{2} \square \mathbf{D}_{\dot{\alpha}}^{m}+\mathbf{C}_{\dot{\alpha}}^{m}-\partial^{m} \mathbf{F}_{\dot{\alpha}}-\frac{i}{\sqrt{2}}\left(\Gamma^{m}\right)_{\dot{\alpha}}^{\beta} \mathbf{I}_{\beta}=0
$$

- multiplying $\left(S^{\dot{\alpha}} e^{-\phi_{6} / 2} c \tilde{c} \partial^{2} \tilde{c}\right)$ :

$$
-\frac{1}{2} \partial^{m} \mathbf{C}_{m \dot{\alpha}}-\mathbf{J}_{\dot{\alpha}}=0
$$

- multiplying $\left(S^{\dot{\alpha}} e^{-\phi_{6} / 2} c \tilde{c} \partial \tilde{c} \partial x^{m}\right)$ :

$$
-\frac{1}{2} \square \mathbf{C}_{m \dot{\alpha}}-\partial_{m} \mathbf{J}_{\dot{\alpha}}=0
$$

- multiplying $\left(S^{\dot{\alpha}} e^{-\phi_{6} / 2} c \tilde{c} \partial \tilde{c} \partial \phi_{6}\right)$ :

$$
+\frac{1}{4} \square \mathbf{G}_{\dot{\alpha}}+\frac{1}{2} \mathbf{J}_{\dot{\alpha}}+\frac{i}{\sqrt{2}}\left(\Gamma^{m}\right)_{\dot{\alpha}}^{\beta} \partial_{m} \mathbf{I}_{\beta}=0
$$

- multiplying $\left(c \tilde{c} \partial \tilde{c} \psi^{m}(\psi S)^{\alpha}\right)$ :

$$
+\frac{1}{2} \square \mathbf{H}_{\alpha}^{m}-\frac{i}{4 \sqrt{2}} \partial_{m} \mathbf{I}_{\alpha}+\frac{i}{8 \times 9 \sqrt{2}}\left(\Gamma_{m}\right)_{\alpha}^{\dot{\beta}}(\not \partial \mathbf{I})_{\dot{\beta}}+\frac{1}{9 \times 4}\left(\Gamma_{m}\right)_{\alpha}^{\dot{\beta}} \mathbf{J}_{\dot{\beta}}=0
$$

- For $1 c, 1 \tilde{c}$

- multiplying $\left(S^{\alpha} e^{\phi_{6} / 2} c \eta \partial \tilde{c}\right)$ :

$$
-\frac{1}{2} \square \mathbf{A}_{\alpha}+\mathbf{B}_{\alpha}+2 \mathbf{I}_{\alpha}-\frac{i}{\sqrt{2}}\left(\Gamma^{m}\right)_{\alpha}^{\dot{\beta}} \partial_{m} \mathbf{F}_{\dot{\beta}}=0
$$

- multiplying $\left(S^{\alpha} e^{\phi_{6} / 2} c \tilde{c} \eta \partial x^{m}\right)$ :

$$
-\partial_{m} \mathbf{B}_{\alpha}+\frac{i}{\sqrt{2}}\left(\Gamma^{n}\right)_{\alpha}^{\dot{\beta}} \partial_{n} \mathbf{C}_{m \dot{\beta}}=0
$$

- multiplying $\left(S^{\alpha} e^{\phi_{6} / 2} c \tilde{c} \eta \mathrm{p}_{m}\right)$ :

$$
-\partial^{m} \mathbf{A}_{\alpha}+\frac{i}{\sqrt{2}}\left(\Gamma^{n}\right)_{\alpha}^{\dot{\beta}} \partial_{n} \mathbf{D}_{\dot{\beta}}^{m}+\frac{i}{2 \sqrt{2}}\left(\Gamma^{m}\right)_{\alpha}^{\dot{\beta}} \mathbf{G}_{\dot{\beta}}-\frac{i 8}{\sqrt{2}} \mathbf{H}_{\alpha}^{m}-\frac{i}{\sqrt{2}} \mathbf{H}_{\beta n}\left(\Gamma^{n}\right)_{\dot{\alpha}}^{\beta}\left(\Gamma^{m}\right)_{\alpha}^{\dot{\alpha}}=0
$$

- multiplying $\left(S^{\alpha} e^{\phi_{6} / 2} c \tilde{c} \partial \eta\right)$ :

$$
\begin{array}{r}
-\mathbf{B}_{\alpha}+3 \mathbf{I}_{\alpha}+\frac{i}{\sqrt{2}}\left(\Gamma^{m}\right)_{\alpha}^{\dot{\beta}} \mathbf{C}_{m \dot{\beta}}-\frac{i}{2 \sqrt{2}}\left(\Gamma^{m}\right)_{\alpha}^{\dot{\beta}} \partial_{m} \mathbf{G}_{\dot{\beta}}+\frac{8 i}{\sqrt{2}} \partial^{m} \mathbf{H}_{\alpha m} \\
+\frac{i}{\sqrt{2}}\left(\Gamma^{n}\right)_{\alpha}^{\dot{\beta}}\left(\Gamma^{m}\right)_{\dot{\beta}}^{\tau} \partial_{n} \mathbf{H}_{\tau m}=0
\end{array}
$$


- multiplying $\left(S^{\alpha} e^{\phi_{6} / 2} c \tilde{c} \eta \partial \phi_{6}\right)$ :

$$
\begin{array}{r}
\frac{1}{2} \mathbf{B}_{\alpha}+4 \mathbf{I}_{\alpha}+\frac{i}{\sqrt{2}}\left(\Gamma^{m}\right)_{\alpha}^{\dot{\beta}} \mathbf{C}_{m \dot{\beta}}-\frac{i}{\sqrt{2}}\left(\Gamma^{m}\right)_{\alpha}^{\dot{\beta}} \partial_{m} \mathbf{G}_{\dot{\beta}}+\frac{8 i}{\sqrt{2}} \partial^{m} \mathbf{H}_{\alpha m} \\
+\frac{i}{\sqrt{2}}\left(\Gamma^{n}\right)_{\alpha}^{\dot{\beta}}\left(\Gamma^{m}\right)_{\dot{\beta}}^{\tau} \partial_{n} \mathbf{H}_{\tau m}=0
\end{array}
$$

- multiplying $\left(\eta c \tilde{c} \psi^{m}(\psi S)^{\dot{\alpha}} e^{\phi_{6} / 2}\right)$ :

$$
\begin{aligned}
+\frac{i}{2 \sqrt{2}}\left[\frac{1}{4} \partial_{m} \mathbf{G}_{\dot{\beta}}-\frac{1}{9 \times 8}\left(\Gamma_{m}\right)_{\dot{\alpha}}^{\tau}(\not \partial \mathbf{G})_{\tau}\right]-\frac{i}{\sqrt{2}}\left[\frac{1}{4} \mathbf{C}_{\dot{\alpha} m}-\frac{1}{9 \times 8}\left(\Gamma_{m}\right)_{\dot{\alpha}}^{\tau}(\not)_{\tau}\right] \\
-\frac{i}{\sqrt{2}}\left[-\frac{1}{4}\left(\Gamma^{n}\right)_{\dot{\alpha}}^{\beta} \partial_{m} \mathbf{H}_{\beta n}-\left(\Gamma^{n}\right)_{\dot{\alpha}}^{\beta} \partial_{n} \mathbf{H}_{\beta m}+\frac{1}{9}\left(\Gamma_{m}\right)_{\dot{\alpha}}^{\beta} \partial^{n} \mathbf{H}_{\beta n}\right] \\
+\frac{1}{36}\left(\Gamma_{m}\right)_{\dot{\alpha}}^{\alpha} \mathbf{B}_{\alpha}-\frac{i}{\sqrt{2}}\left[\frac{1}{9 \times 8}\left(\Gamma_{m}\right)_{\dot{\alpha}}^{\beta}\left(\Gamma^{l}\right)_{\beta}^{\dot{\beta}}\left(\Gamma^{p}\right)_{\dot{\beta}}^{\tau} \partial_{l} \mathbf{H}_{\tau p}\right]=0
\end{aligned}
$$

These 14 equations of motion are all invariant under the following 10 gauge transformations:

$$
\begin{aligned}
\delta \mathbf{A}_{\alpha} & =+\frac{i}{\sqrt{2}}\left(\Gamma^{m}\right)_{\alpha}^{\dot{\beta}} \partial_{m} \lambda_{\dot{\beta}}+2 \mu_{\alpha} \\
\delta \mathbf{B}_{\alpha} & =+\frac{i}{\sqrt{2}}\left(\Gamma^{m}\right)_{\alpha}^{\dot{\beta}} \partial_{m} \omega_{\dot{\beta}} \\
\delta \mathbf{I}_{\alpha} & =\frac{1}{2} \square \mu_{\alpha} \\
\delta \mathbf{H}_{\alpha}^{m} & =\frac{1}{9 \times 4}\left(\Gamma^{m}\right)_{\alpha}^{\dot{\beta}} \omega_{\dot{\beta}}+\frac{i}{4 \sqrt{2}} \partial_{m} \mu_{\alpha}-\frac{i}{8 \times 9 \sqrt{2}}\left(\Gamma_{m}\right)_{\alpha}^{\dot{\beta}}(\not \partial \mu)_{\dot{\beta}} \\
\delta \mathbf{C}_{m \dot{\alpha}} & =\partial_{m} \omega_{\dot{\alpha}} \\
\delta \mathbf{D}_{\dot{\alpha}}^{m} & =\partial_{m} \lambda_{\dot{\alpha}}-\frac{i}{\sqrt{2}}\left(\Gamma^{m}\right)_{\dot{\alpha}}^{\beta} \mu_{\beta} \\
\delta \mathbf{E}_{\dot{\alpha}}^{A} & =0 \\
\delta \mathbf{F}_{\dot{\alpha}} & =-\frac{1}{2} \square \lambda_{\dot{\alpha}}+\omega_{\dot{\alpha}} \\
\delta \mathbf{G}_{\dot{\alpha}} & =\omega_{\dot{\alpha}}-\frac{2 i}{\sqrt{2}}\left(\Gamma^{m}\right)_{\dot{\alpha}}^{\beta} \partial_{m} \mu_{\beta} \\
\delta \mathbf{J}_{\dot{\alpha}} & =-\frac{1}{2} \square \omega_{\dot{\alpha}}
\end{aligned}
$$

We determined the basic content of ghost number 2 BRST cohomology; all equations of motion have been written between (3.5) and (3.18). This set is highly redundant, and the next step is to use (3.19) to stablish the independent field equations.

\subsection{Gauge-fixing and independent equations of motion}

In order to find the independent set of equations of motion, we begin by fixing algebraic gauge conditions and solving auxiliary field equations. Let us gauge-fix $\mathbf{A}$ and $\mathbf{F}$ to zero 
using the parameters $\mu$ and $\omega$, that is, we choose $\mu=-\mathbf{A}$ and $\omega=-\mathbf{F}$ so that the residual gauge parameters $\mu^{\prime}$ and $\omega^{\prime}$ must satisfy:

$$
\mu_{\alpha}^{\prime}+\frac{i}{2 \sqrt{2}}\left(\Gamma^{m}\right)_{\alpha}^{\dot{\beta}} \partial_{m} \lambda_{\dot{\beta}}=0
$$

and

$$
\omega_{\dot{\alpha}}^{\prime}-\frac{1}{2} \square \lambda_{\dot{\alpha}}=0
$$

After this gauge fixing, the following auxiliary field conditions can be imposed:

$$
\begin{aligned}
\mathbf{G}_{\dot{\alpha}}^{m} & =0, \\
\mathbf{B}_{\alpha} & =-2 \mathbf{I}_{\alpha}, \\
\mathbf{C}_{\dot{\alpha}}^{m} & =+\frac{1}{2} \square \mathbf{D}_{\dot{\alpha}}^{m}+\frac{i}{\sqrt{2}}\left(\Gamma^{m} \mathbf{I}\right)_{\dot{\alpha}}, \\
\mathbf{J}_{\dot{\alpha}} & =-\frac{1}{4} \square \partial_{m} \mathbf{D}_{\dot{\alpha}}^{m}-\frac{i}{2 \sqrt{2}}(\not \mathbf{I})_{\dot{\alpha}}, \\
\mathbf{H}_{\alpha}^{m} & =\frac{1}{8} \not \partial_{\alpha}^{\dot{\beta}} \mathbf{D}_{\dot{\beta}}^{m}-\frac{1}{18 \times 8}\left(\Gamma^{m} \Gamma_{n} \not \partial\right)_{\alpha}^{\dot{\beta}} \mathbf{D}_{\dot{\beta}}^{n} .
\end{aligned}
$$

At this point it is already clear that there only remains two independent fields given by $\mathbf{D}_{\dot{\alpha}}^{m}$ and $\mathbf{I}_{\alpha}$. Moreover, the only remaining gauge parameter is $\lambda$. We leave the gluino field $\mathbf{E}_{\dot{\beta}}^{a}$ out of the discussion since its equation of motion is already the Dirac equation and it has no gauge transformations.

Finally, the following set of 3 equations,

$$
\begin{aligned}
\frac{i}{\sqrt{2}} \partial^{m} \mathbf{I}_{\alpha} & =\square\left(\frac{1}{4} \not \not_{\alpha}^{\dot{\beta}} \mathbf{D}_{\dot{\beta}}^{m}-\frac{1}{12}\left(\Gamma^{m}\right)_{\alpha}^{\dot{\beta}} \partial_{n} \mathbf{D}_{\dot{\beta}}^{n}\right) \\
2 \partial_{m} \mathbf{D}_{\dot{\alpha}}^{m}+\left(\Gamma_{n} \Gamma_{p}\right)_{\dot{\alpha}}^{\dot{\beta}} \partial^{n} \mathbf{D}_{\dot{\beta}}^{p} & =0 \\
\partial_{\alpha}^{\dot{\alpha}} \mathbf{E}_{\dot{\alpha}}^{a} & =0
\end{aligned}
$$

with the corresponding gauge transformations:

$$
\begin{aligned}
\delta \mathbf{D}_{\dot{\alpha}}^{m} & =\frac{3}{4} \partial^{m} \lambda_{\dot{\alpha}}-\frac{1}{4}\left(\Gamma^{m n}\right)_{\dot{\alpha}}^{\dot{\beta}} \partial_{n} \lambda_{\dot{\beta}} \\
\delta \mathbf{I}_{\alpha} & =-\frac{i}{4 \sqrt{2}} \not_{\alpha}^{\dot{\beta}} \square \lambda_{\dot{\beta}}
\end{aligned}
$$

defines the spectrum of the theory.

Gauge-invariant description. Consider the following field redefinitions:

$$
\begin{aligned}
\mathbf{d}_{\dot{\alpha}}^{m} & =\mathbf{D}_{\dot{\alpha}}^{m}-\frac{1}{6}\left(\Gamma^{m}\right)_{\dot{\alpha}}^{\alpha} \boldsymbol{D}_{\alpha} \\
\mathbf{i}_{\alpha} & =+\frac{i 4}{\sqrt{2}} \mathbf{I}_{\alpha}+\frac{1}{6} \square \not D_{\alpha}
\end{aligned}
$$

such that our gauge transformations are mapped to

$$
\delta \mathbf{d}_{\dot{\alpha}}^{m}=\partial^{m} \lambda_{\dot{\alpha}} \quad \text { and } \quad \delta \mathbf{i}_{\alpha}=0 .
$$


The gauge-invariant object is then naturally defined as:

$$
\mathbf{F}_{m n \dot{\alpha}}=\partial_{m} \mathbf{d}_{n \dot{\alpha}}-\partial_{n} \mathbf{d}_{m \dot{\alpha}}
$$

which allows us to write the equations of motion in the following form:

$$
\partial_{m} \mathbf{i}_{\alpha}=\square \mathbf{F}_{m \alpha}
$$

and

$$
(\mathbf{F})_{\dot{\alpha}}=0
$$

where

$$
\mathbf{F}_{m \alpha} \equiv\left(\Gamma^{n}\right)_{\alpha}^{\dot{\alpha}} \mathbf{F}_{m n \dot{\alpha}}=\left(\not \partial \mathbf{d}_{m}-\partial_{m} \not \mathbf{d}\right)_{\alpha} .
$$

In the formulation of free higher-spin theories $\mathbf{F}_{m}$ is called Fronsdal tensor [4], it is the analog of the Ricci curvature in spin 2 formulation.

This section started with the most general ghost number 2 picture $-1 / 2$ vertex operator. Then we obtained all equations of motion from the BRST method together with all gauge transformations parametrized by ghost number 1 picture $-1 / 2$ vertex operators. By fixing some of this gauge freedom, we have found a independent set of equations of motion that can be parametrized by Fronsdal fields. The next natural step is to write the spacetime action that gives the dynamics of this system.

\section{Action}

The kinetic term of the ambitwistor string field theory was defined in [3]:

$$
S[V]=\left\langle I \circ V^{(-3 / 2)}(0) \partial c Q V^{(-1 / 2)}(0)\right\rangle,
$$

where $V^{-1 / 2}$ is the vertex operator (3.2) introduced in the previous section, an element of the small Hilbert space that is also constrained to satisfy $L_{0} V=b_{0} V=0$. The RNS string has one additional feature: the picture number. It is necessary to saturate the background charge of supermoduli space to -2 , and that is why we need a string field with picture $-1 / 2, V^{-1 / 2}$, together with a string field with picture $-3 / 2, V^{-3 / 2}$. We define picture raising, $Z$, and picture lowering, $Y$, by the following expressions:

$$
\begin{aligned}
Z & =c \partial \xi+e^{\phi_{6}} \mathrm{p}_{m} \psi^{m}-\partial\left(e^{2 \phi_{6}} \eta \tilde{b}\right)-e^{2 \phi_{6}} \partial \eta \tilde{b}, \\
Y(z) & =\tilde{c} \partial \xi e^{-2 \phi_{6}},
\end{aligned}
$$

so that we can obtain $V^{-3 / 2}$ from $V^{-1 / 2}$ via

$$
V^{-3 / 2}(z)=\frac{1}{2 \pi i} \oint \frac{d w}{(w-z)} Y(w) V^{-1 / 2}(z) .
$$

Using the auxiliary gauge-fixing conditions imposed on the previous section, we obtain

$$
\begin{aligned}
V^{-3 / 2}= & +\tilde{c} \partial \tilde{c} S^{\alpha} e^{-3 \phi_{6} / 2} \mathbf{B}_{\alpha}-c \tilde{c} \partial \tilde{c} \partial \xi S^{\dot{\alpha}} e^{-5 \phi_{6} / 2} \partial x^{m} \mathbf{C}_{m \dot{\alpha}}-c \tilde{c} \partial \tilde{c} \partial \xi S^{\dot{\alpha}} e^{-5 \phi_{6} / 2} \mathrm{p}_{m} \mathbf{D}_{\dot{\alpha}}^{m} \\
& -c \tilde{c} \partial \tilde{c} \partial \xi S^{\dot{\alpha}} e^{-5 \phi_{6} / 2} j^{a} \mathbf{E}_{\dot{\alpha}}^{a}-c \tilde{c} \partial \tilde{c} \partial \xi \psi_{m}(\psi S)^{\alpha} e^{-5 \phi_{6} / 2} \mathbf{H}_{\alpha}^{m} \\
& -\frac{1}{2} c \tilde{c} \partial \tilde{c} \partial \xi \partial^{2} \tilde{c} \partial^{2} \xi S^{\alpha} e^{-7 \phi_{6} / 2} \mathbf{I}_{\alpha}
\end{aligned}
$$


The composition $I \circ V^{-3 / 2}$ is the BPZ conjugate of the picture $-3 / 2$ field with $I=-1 / z$. We should be careful when computing the conformal transformation $I \circ V^{-3 / 2}$ because $V^{-1 / 2}$ is not primary. From the OPE with the stress-energy tensor

$$
T(z) V^{-1 / 2}(0) \sim z^{-3} S^{\dot{\alpha}} e^{-\phi_{6} / 2} c \tilde{c}\left(\frac{1}{2} \partial_{m} \mathbf{D}_{\dot{\alpha}}^{m}+\mathbf{F}_{\dot{\alpha}}-\frac{3}{8} \mathbf{G}_{\dot{\alpha}}-\frac{9}{4} \mathbf{H}_{\dot{\alpha}}\right)+\cdots
$$

we obtain a cubic pole contribution that changes the finite conformal transformation to

$$
I \circ V=\left[V(I(z))+\frac{1}{2} \frac{I^{\prime \prime}(z)}{\left[I^{\prime}(z)\right]^{2}} \#(I(z))\right],
$$

where \# is cubic pole coefficient. Even after the auxiliary conditions are imposed we still have non-primary contributions that must be taken into account.

To calculate the free action, we fix the normalization $\left\langle c \partial c \partial^{2} c \tilde{c} \partial \tilde{c} \partial^{2} \tilde{c} e^{-2 \phi_{6}}\right\rangle=4$, then the correlation function (4.1) gives the following gauge-invariant action:

$$
S_{R}=-\int d^{10} x\left[\frac{1}{2} \mathbf{d}^{m \alpha} \square\left(\mathbf{F}_{m \alpha}-\frac{1}{2}\left(\gamma_{m}\right)_{\alpha \beta} \mathbf{F}^{\beta}\right)+\frac{1}{2}(\mathbf{F})^{\alpha} \mathbf{i}_{\alpha}-\frac{i}{2} \operatorname{Tr}(\mathbf{E} \not \mathbf{E})\right] .
$$

In this expression we used the symmetric gamma matrices $\left(\gamma_{\alpha \beta}^{m}, \gamma_{m}^{\alpha \beta}\right)$ defined in appendix A. When using these symmetric matrices, the charge conjugation is used to eliminate all dotted indices; different chiralities are just represented by upper and lower indices, i.e. $\left(C^{\alpha \dot{\alpha}} \mathbf{d}_{\dot{\alpha}}^{m}=\mathbf{d}^{m \alpha}\right)$.

We have written a non-unitary action that gives the equations of motion obtained in (3.28). It closely resembles the gauge-invariant formulation of spin $3 / 2$, the difference being the presence of more derivatives. Let us proceed and study the supersymmetry of this non-unitary system.

\section{Supersymmetry}

Let us define the supersymmetry generator as

$$
\mathbf{Q}_{\alpha}^{-1 / 2}=\frac{1}{2 \pi i} \oint \mathrm{d} z S_{\alpha} e^{-\phi_{6} / 2}
$$

Notice that it carries picture, which means that supersymmetry algebra only closes on-shell. We need the picture $1 / 2$ supersymmetric charge:

$$
\mathbf{Q}_{\alpha}^{1 / 2}=\frac{1}{2 \pi i} \oint \mathrm{d} z\left[i \mathrm{p}_{m}\left(\gamma^{m}\right)_{\alpha \beta} S^{\beta} e^{\phi_{6} / 2}+\tilde{b} \eta S_{\alpha} e^{3 \phi_{6} / 2}\right],
$$

to obtain $\left\{Q_{\alpha}^{-1 / 2}, Q_{\beta}^{1 / 2}\right\}=2 \gamma_{\alpha \beta}^{m} \mathrm{p}_{m}$. In practice, supersymmetry transformations are written up to equations of motion. One also needs to choose a GSO sector to have well-defined supersymmetry transformations, otherwise there will be branch cuts. Given the generator (5.1), we need use the $\mathrm{GSO}(+)$ vertex operator. 


\subsection{Supersymmetry transformations of NS and R sectors}

The Neveu-Schwarz vertex operator in picture -1 was written in [3]:

$$
\begin{aligned}
V_{\mathrm{NS}}^{-1}= & e^{-\phi_{6}} c \tilde{c}\left[\left(G_{(m n)}^{(1)}+B_{[m n]}^{(1)}\right) \mathrm{p}^{m} \psi^{n}+\left(G_{(m n)}^{(2)}+B_{[m n]}^{(2)}\right) \partial x^{m} \psi^{n}+C_{m n p} \psi^{m} \psi^{n} \psi^{p}+\mathrm{j}^{a} \psi^{m} A_{m}^{a}\right] \\
& +e^{-\phi_{6}} c \tilde{c} \partial \psi^{m} A_{m}^{(4)}+\partial \phi_{6} e^{-\phi_{6}} c \tilde{c} A_{m}^{(3)} \psi^{m}+\partial \xi e^{-2 \phi_{6}} \partial^{2} \tilde{c} \tilde{c} c S^{(4)}+\eta c S^{(1)}+\partial \xi e^{-2 \phi_{6}} \partial^{2} c c \tilde{c} S^{(2)} \\
& +\ldots
\end{aligned}
$$

where ... depends only on the previous fields. In [3], the fields $\left(B_{m n}^{(1)}, A_{m}^{(3)}, A^{(4)}, S^{(1)}, S^{(2)}\right)$ of (5.3) were gauged to zero. If we choose to keep this gauge, we must observe that in general supersymmetry does not preserve a given gauge condition. Therefore when calculating supersymmetry transformations, we have to choose the gauge parameter $\Lambda$ :

$$
\delta_{\zeta} V_{\mathrm{NS}}^{-1}=\left[\zeta \mathbf{Q}^{-1 / 2}, V_{R}^{-1 / 2}\right]+\left[Q_{B R S T}, \Lambda^{-1}\right]
$$

which is a vertex operator of ghost number 1 and picture -1 , to ensure that $\delta_{\zeta}\left(B_{m n}^{(1)}, A_{m}^{(3)}, A^{(4)}, S^{(1)}, S^{(2)}\right)$ all give zero. In the transformations below, the contributions of $\mathbf{H}$ are due to the gauge-fixing of these auxiliary fields:

$$
\begin{aligned}
\delta_{\zeta} G_{m n}^{(1)} & =2\left(\zeta \gamma_{(m} \mathbf{D}_{n)}\right) \\
\delta_{\zeta} G_{m n}^{(2)} & =\frac{2}{5}\left(\zeta \gamma_{(n} \mathbf{C}_{m)}\right)-\frac{48}{5} \partial_{(n} \zeta \mathbf{H}_{m)} \\
\delta_{\zeta} B_{m n}^{(2)} & =-4\left(\zeta \gamma_{[n} \mathbf{C}_{m]}\right)-\frac{48}{5}\left(\zeta \partial_{[m} \mathbf{H}_{n]}\right) \\
\delta_{\zeta} C_{m n p} & =\frac{3}{2} \partial_{[p}\left(\zeta \gamma_{m} \mathbf{D}_{n]}\right)-24\left(\zeta \gamma_{[n p} \mathbf{H}_{m]}\right)+6\left(\zeta \gamma_{m n p} \mathbf{H}\right)
\end{aligned}
$$

and using the field redefinitions of [3]:

$$
h_{m n}=G_{m n}^{(1)}+\frac{1}{4} \eta_{m n} h_{r}^{r}, \quad t=\frac{1}{4} \square h_{m}^{m}+G_{m}^{m(2)} \quad \text { and } \quad B_{m n}^{(2)}=B_{m n}
$$

we arrive at

$$
\begin{aligned}
\delta_{\zeta} h_{m n} & =2 \zeta \gamma_{(m} \mathbf{d}_{n)} \\
\delta_{\zeta} t & =\zeta \mathbf{i} \\
\delta_{\zeta} C_{m n p} & =-3\left(\zeta \gamma_{t[m n} \mathbf{F}_{p]}^{t}\right)-3\left(\zeta \gamma_{[m} \mathbf{F}_{n p]}\right) \\
\delta_{\zeta} B_{m n} & =-2 \square\left(\zeta \gamma_{[m} \mathbf{d}_{n]}\right)-\left(\zeta \gamma_{m n} \mathbf{i}\right)+\frac{1}{6}\left(\zeta \gamma_{m n} \partial_{p} \mathbf{F}^{p}\right) \\
\delta_{\zeta} A_{m}^{a} & =\frac{i}{2}\left(\zeta \gamma_{m} \mathbf{E}^{a}\right) .
\end{aligned}
$$

The term $\left(\zeta \gamma_{m n} \partial_{p} \mathbf{F}^{p}\right)$ is zero if we use the equation of motion $=0$, and so could not have been obtained from the supersymmetry generator (5.1). This term was added by hand in order to make the action invariant under supersymmetry. 
For the Ramond sector the same can be done if we use instead the picture $+1 / 2$ supersymmetry generator (5.2):

$$
\begin{aligned}
\delta_{\zeta} \mathbf{d}_{m}^{\alpha} & =+\left(\gamma^{r s} \zeta\right)^{\alpha} \partial_{s} h_{m r}-2\left(\gamma^{n p} \zeta\right)^{\alpha} C_{m n p}+\frac{1}{3}\left(\gamma_{m n p s} \zeta\right) C^{n p s} \\
\delta_{\zeta} \mathbf{i}_{\alpha} & =2(\zeta \not)_{\alpha} t-\left(\gamma^{m n p} \zeta\right)_{\alpha} H_{m n p}+\frac{1}{3}\left(\gamma^{m n p} \zeta\right)_{\alpha} \square C_{m n p} \\
\delta_{\zeta} \mathbf{E}^{a \beta} & =-\frac{1}{4} F_{m n}\left(\gamma^{m n} \zeta\right)^{\beta}
\end{aligned}
$$

At this point, we have obtain the supersymmetry transformations of both NS and R system for the independent fields of the theory in equations (5.10) to (5.9). Let us proceed and check that indeed the total $G S O(+)$ action is supersymmetric invariant.

\subsection{Supersymmetry invariance of the action}

The action that describes the Neveu-Schwarz sector is

$$
\begin{aligned}
S_{\mathrm{NS}}=-\int d^{10} x\left[\frac{1}{2} h^{m n} \square\right. & \left(R_{m n}-\frac{1}{2} \eta_{m n} R\right)-t R+\frac{1}{4} \operatorname{Tr}\left(F^{m n} F_{m n}\right) \\
& \left.-C^{m n p} H_{m n p}+\frac{1}{2} C^{m n p}\left(\square C_{m n p}-\frac{1}{2} \partial_{[p} \partial^{r} C_{m n] r}\right)\right]
\end{aligned}
$$

where $H_{m n p}$ is the field strength for $B_{m n}$ and $R_{m n}$ is the Ricci tensor. This expression is equivalent to the action written in equation (4.13) of [3] if we shift $t$ by $t \mapsto t+R^{2}$. The equations of motion derived from (5.18) are $^{1}$

$$
\begin{aligned}
\square R_{m n}-\partial_{m} \partial_{n} t & =0, & R=0, & \square C_{m n p}-H_{m n p} & =0, \\
\partial^{m} C_{m n p} & =0, & \text { and } & \partial_{m} F^{m n} & =0 .
\end{aligned}
$$

Now, the Ramond sector is described by equation (4.8):

$$
S_{R}=-\int d^{10} x\left[\frac{1}{2} \mathbf{d}^{m \alpha} \square\left(\mathbf{F}_{m \alpha}-\frac{1}{2}\left(\gamma_{m}\right)_{\alpha \beta} \mathbf{F}^{\beta}\right)+\frac{1}{2}(\mathbf{F})^{\alpha} \mathbf{i}_{\alpha}-\frac{i}{2} \operatorname{Tr}(\mathbf{E} \not \mathbf{E})\right],
$$

from which we obtain the following set of equations of motion - (3.28):

$$
\partial_{m} \mathbf{i}_{\alpha}=\square \mathbf{F}_{m \alpha}, \quad \not \mathbf{F}^{\alpha}=0 \quad \text { and } \quad i \not \partial_{\alpha \beta} \mathbf{E}^{a \beta}=0 .
$$

From now on, we leave the Yang-Mills system out of the discussion because its supersymmetry transformations and action are already standard. For later use, let us write the

\footnotetext{
${ }^{1}$ Notice that we use the fact

$$
\left(\delta h_{m n}\right) \square\left(R_{m n}-\frac{1}{2} \eta_{m n} R\right)=h_{m n} \square \delta\left(R_{m n}-\frac{1}{2} \eta_{m n} R\right)+\text { total derivative } .
$$
}


supersymmetry transformation for all field strengths:

$$
\begin{aligned}
\delta_{\zeta} R_{m n}= & \left(\zeta \partial_{(m} \mathbf{F}_{n)}\right)+\left(\zeta \gamma_{(m} \partial^{p} \mathbf{F}_{n) p}\right) \\
\delta_{\zeta} H_{m n p}= & 3 \square\left(\zeta \gamma_{[m} \mathbf{F}_{n p]}\right)-3\left(\zeta \gamma_{[m n} \partial_{p]} \mathbf{i}\right)+\frac{1}{2}\left(\zeta \gamma_{[m n} \partial_{p]} \partial_{\ell} \mathbf{F}^{\ell}\right) \\
\delta_{\zeta} \mathbf{F}_{m n}^{\alpha}= & -2\left(\gamma^{r s} \zeta\right)^{\alpha} R_{m r s n}+4\left(\gamma^{r p} \zeta\right)^{\alpha} \partial_{[n} C_{m] r p}-\frac{2}{3}\left(\partial_{[n} \gamma_{m] r p s} \zeta\right)^{\alpha} C^{r p s} \\
\delta_{\zeta} \mathbf{F}_{m \alpha}= & +2\left(\gamma^{n} \zeta\right)_{\alpha} R_{m n}-2\left(\gamma^{l n p} \zeta\right)_{\alpha} \partial_{l} C_{m n p}+\frac{1}{3}\left(\gamma_{l m n p s} \zeta\right)_{\alpha} \partial^{l} C^{n p s} \\
& +4\left(\gamma^{n} \zeta\right)_{\alpha} \partial^{p} C_{m n p}-\left(\gamma_{m p s} \zeta\right)_{\alpha} \partial_{n} C^{n p s} \\
\delta \mathbf{F}^{\beta}= & 2 \zeta^{\beta} R-6\left(\gamma^{n p} \zeta\right)^{\beta} \partial^{m} C_{n p m}
\end{aligned}
$$

\subsection{Supersymmetry for $\left(h_{m n}, t, \mathrm{i}, \mathrm{d}\right)$}

Let us consider the system:

$$
\begin{aligned}
\mathbf{S}=-\int d^{10} x\left(\frac{1}{2} h^{m n}\right. & \square\left(R_{m n}-\frac{1}{2} \eta_{m n} R\right)-t R \\
& \left.+\frac{1}{2} \mathbf{d}^{m \alpha} \square\left(\mathbf{F}_{m \alpha}-\frac{1}{2}\left(\gamma_{m}\right)_{\alpha \beta} \mathbf{F}^{\beta}\right)+\frac{1}{2}(\mathbf{F})^{\alpha} \mathbf{i}_{\alpha}\right)
\end{aligned}
$$

such that the

$\boldsymbol{S}_{\mathrm{NS}}$ variation is given by

$$
\begin{aligned}
\delta_{\zeta}(-t R) & =-\zeta^{\alpha} \mathbf{i}_{\alpha} R-2 t \zeta^{\alpha} \partial_{p} \mathbf{F}_{\alpha}^{p} \\
\delta_{\zeta}\left[\frac{1}{2} h^{m n} \square\left(R_{m n}-\frac{1}{2} \eta_{m n} R\right)\right] & =2\left(\zeta \gamma^{m} \mathbf{d}^{n}\right) \square\left(R_{m n}-\frac{1}{2} \eta_{m n} R\right)
\end{aligned}
$$

and the

$\boldsymbol{S}_{\boldsymbol{R}}$ variation is given by

$$
\begin{aligned}
\delta_{\zeta}\left(\frac{1}{2} \mathbf{d}^{m \alpha} \square\left(\mathbf{F}_{m \alpha}-\frac{1}{2}\left(\gamma_{m}\right)_{\alpha \beta} \mathbf{F}^{\beta}\right)\right) & =2 \mathbf{d}^{m \alpha} \square\left(\left(\zeta \gamma^{n}\right)_{\alpha} R_{m n}-\frac{1}{2}\left(\zeta \gamma_{m}\right)_{\alpha} R\right) \\
& =-2\left(\zeta \gamma^{n} \mathbf{d}^{m}\right) \square\left(R_{m n}-\frac{1}{2} \eta_{m n} R\right) \\
\delta_{\zeta}\left(\frac{1}{2}(\mathbf{F})^{\alpha} \mathbf{i}_{\alpha}\right) & =\zeta^{\alpha} R \mathbf{i}_{\alpha}+(\mathbf{F})^{\alpha} \not \partial_{\alpha \beta} \zeta^{\beta} t \\
& =\zeta^{\alpha} \mathbf{i}_{\alpha} R+2 \zeta^{\alpha}\left(\partial_{p} \mathbf{F}_{\alpha}^{p}\right) t+\partial(\ldots)
\end{aligned}
$$

where we have used (5.22) and $(\not \partial \zeta)=2\left(\zeta \partial_{p} \mathbf{F}^{p}\right)$. It is clear that the sum of all terms cancels and invariance of this system is stablished. 


\subsection{Supersymmetry for $\left(H_{m n p}, C_{m n p}, \mathrm{~d}_{m}^{\alpha}, \mathrm{i}_{\alpha}\right)$}

It remains for consideration the following system:

$$
\begin{aligned}
\mathbf{S}=-\int d^{10} x\left(-C^{m n p} H_{m n p}\right. & +\frac{1}{2} C^{m n p}\left(\square C_{m n p}-\frac{1}{2} \partial_{[p} \partial^{r} C_{m n] r}\right) \\
+ & \left.\frac{1}{2} \mathbf{d}^{m \alpha} \square\left(\mathbf{F}_{m \alpha}-\frac{1}{2}\left(\gamma_{m}\right)_{\alpha \beta} \mathbf{F}^{\beta}\right)+\frac{1}{2}(\mathbf{F})^{\alpha} \mathbf{i}_{\alpha}\right)
\end{aligned}
$$

In order to check supersymmetric invariance we have to gather all independent combination of gamma matrices $\left(\gamma^{m}, \gamma^{m n}, \gamma^{m n p}, \gamma^{m n p p}, \gamma^{m n p q r}\right)$. So consider the

\section{$S_{\mathrm{NS}}$ variation:}

$$
\begin{gathered}
\delta_{\zeta}\left(-C^{m n p} H_{m n p}\right)=+3\left[\left(\zeta \gamma_{t m n} \mathbf{F}_{p}^{t}\right)+\left(\zeta \gamma_{m} \mathbf{F}_{n p}\right)\right] H^{m n p}-3\left(\zeta \gamma_{m} \mathbf{F}_{n p}\right) \square C^{m n p} \\
-3\left(\zeta \gamma_{m n} \mathbf{i}\right) \partial_{p} C^{m n p}-\frac{1}{2}\left(\zeta \gamma_{m n} \partial_{p} \mathbf{F}^{p}\right) \partial_{p} C^{m n p}+\partial(\ldots) \\
\delta_{\zeta}\left[\frac{1}{2} C^{m n p}\left(\square C_{m n p}-\frac{1}{2} \partial_{[p} \partial^{r} C_{m n] r}\right)\right]= \\
-3\left[\left(\zeta \gamma_{t m n} \mathbf{F}_{p}^{t}\right)+\left(\zeta \gamma_{m} \mathbf{F}_{n p}\right)\right] \square C^{m n p} \\
+\frac{1}{2}\left[\left(\zeta \gamma_{m n} \partial^{p} \mathbf{F}_{p}\right)+\left(\zeta \gamma_{m} \partial_{n} \mathbf{F}\right)\right] \partial_{r} C^{m n r}+\partial(\ldots)
\end{gathered}
$$

and the

\section{$S_{R}$ variation:}

$$
\begin{aligned}
\delta_{\zeta}\left(\frac{1}{2} \mathbf{F i}\right)= & -3\left(\gamma^{n p} \zeta\right)^{\beta} \partial^{m} C_{n p m} \mathbf{i}_{\beta}-\frac{1}{2} \mathbf{F}^{\alpha}\left(\gamma^{m n p} \zeta\right)_{\alpha} H_{m n p}+\frac{1}{6} \mathbf{F}^{\alpha}\left(\gamma^{m n p} \zeta\right)_{\alpha} \square C_{m n p} \\
= & +3\left(\zeta \gamma^{n m} \mathbf{i}\right) \partial^{p} C_{n m p} \\
& -\left[\frac{1}{6}\left(\zeta \gamma^{m n p t s} \mathbf{F}_{t s}\right) \square C_{m n p}-\left(\zeta \gamma^{t m n} \mathbf{F}_{t}{ }^{p}\right) \square C_{m n p}-\left(\zeta \gamma^{m} \mathbf{F}^{n p}\right) \square C_{m n p}\right] \\
& +\left[\frac{1}{2}\left(\zeta \gamma^{m n p t s} \mathbf{F}_{t s}\right) H_{m n p}-3\left(\zeta \gamma^{t m n} \mathbf{F}_{t}^{p}\right) H_{m n p}-3\left(\zeta \gamma^{m} \mathbf{F}^{n p}\right) H_{m n p}\right] \\
\delta_{\zeta}\left(\frac{1}{2} \mathbf{d}_{m}^{\alpha} \square\right. & \left.\left(\mathbf{F}_{\alpha}^{m}-\frac{1}{2}\left(\gamma^{m}\right)_{\alpha \beta} \mathbf{F}^{\beta}\right)\right)= \\
= & \left(-2\left(\gamma^{n p} \zeta\right)^{\alpha} C_{m n p}+\frac{1}{3}\left(\gamma_{m n p s} \zeta\right)^{\alpha} C^{n p s}\right) \square\left(\mathbf{F}_{\alpha}^{m}-\frac{1}{2}\left(\gamma^{m}\right)_{\alpha \beta} \mathbf{F}^{\beta}\right) \\
= & +2\left(\mathbf{F}_{l}{ }^{m} \gamma^{l n p} \zeta\right) \square C_{m n p}-4\left(\mathbf{F}^{m n} \gamma^{p} \zeta\right) \square C_{m n p} \\
& -\frac{1}{3}\left(\mathbf{F}_{l m} \gamma^{l m n p s} \zeta\right) \square C_{n p s}-\left(\mathbf{F}_{m}^{n} \gamma^{p s m} \zeta\right) \square C_{n p s} \\
& -\left[\frac{1}{6}\left(\zeta \gamma^{m n p t s} \mathbf{F}_{t s}\right) \square C_{m n p}-\left(\zeta \gamma^{t m n} \mathbf{F}_{t}^{p}\right) \square C_{m n p}-\left(\zeta \gamma^{m} \mathbf{F}^{n p}\right) \square C_{m n p}\right]
\end{aligned}
$$

Recall that the $\gamma^{m n p q r}$ is symmetric and $\gamma^{m n p}$ is antisymmetric under the spinor indices. Gathering all independent terms we confirm the system is supersymmetric. 


\section{Acknowledgments}

We would like to thank Oliver Schlotterer and Nathan Berkovits for useful discussions, Andrei Mikhailov and Nathan Berkovits for helpful comments on the manuscript. ML thanks FAPESP grant 2016/16824-0 for financial support and HF would like to thank CAPES grant 33015015001P7 for financial support.

\section{A Ramond sector, cocycles and Gamma matrices}

Spinor indices in 10 dimensions can be distinguished between chiral and anti-chiral. We denote chiral indices by undotted greek letters, $\alpha$, while anti-chiral indices are represented by dotted greek letters, $\dot{\alpha}$. Both run from 1 to 16 . Spinor indices are 5-dimensional vector representations of $u(5)$ :

$$
\dot{\alpha}=\frac{1}{2}\left(\begin{array}{l}
----- \\
---++ \\
-++++
\end{array}\right) \quad \text { and } \beta=\frac{1}{2}\left(\begin{array}{c}
+++++ \\
+++-- \\
+----
\end{array}\right),
$$

where an anti-chiral index, $\dot{\alpha}$, must have an even number of plus signs, and a chiral index, $\beta$, must have an odd number of plus signs. Each of these combinations has 16 independent components represented as $\mathbf{1 6}=\mathbf{1}+\mathbf{1 0}+\mathbf{5}$.

\section{A.1 The Ramond sector}

The Ramond sector of the Ambitwistor string is defined by the antiperiodic boundary conditions of $\psi^{m}$ :

$$
\psi^{m}\left(e^{2 \pi i} z\right)=-\psi^{m}(z) .
$$

We follow [5] and implement these boundary conditions via spin fields. That is, we have a conformal primary $S(z)$ that twists a periodic $\psi$ :

$$
\psi^{m}\left(z+(w-z) e^{2 \pi i}\right) S(z)=-\psi^{m}(w) S(z) .
$$

This implies that a state $|\alpha\rangle$ created from the vacuum $|0\rangle$ via

$$
|\alpha\rangle=S^{\alpha}(0)|0\rangle
$$

should transform as a spacetime spinor. Notice that, due to the presence of $S$ forcing $\psi$ to be in the Ramond sector, this state must belong to an irreducible representation of the zero-mode Clifford algebra of $\psi^{m}:\left\{\psi_{0}^{m}, \psi_{0}^{n}\right\}=\eta^{m n}$, which implies

$$
\psi_{0}^{m}|\alpha\rangle=\frac{1}{\sqrt{2}} \Gamma_{\dot{\beta}}^{m \alpha}|\dot{\beta}\rangle .
$$

\section{A.2 Bosonization and cocycles}

Because $S^{\alpha}$ twists the boundary conditions of $\psi^{m}$, the system is not free and OPE's are difficult to compute. Bosonization is a technique that allows us to deal with free fields only. Bosonization assigns for a pair of complex fermions one chiral boson, which means that we have to break manifest $s o(10)$ invariance down to $u(5)$. 
Spin fields. The bosonization of spin fields is given by

$$
S^{\alpha}(z)=\exp (\alpha \cdot \phi(z)) c_{\alpha}
$$

where $\alpha$ is a chiral spinor index. The same expression is valid for anti-chiral spin fields by just replacing $\alpha$ for $\dot{\alpha}$. The factor $c_{\alpha}$ is a cocycle phase that guarantees the correct anticommutation relations.

Cocycles. The anticommuting fermionic algebra is reproduced in the bosonic system via the Baker-Campbell-Hausdorff formula: ${ }^{2}$

$$
e^{\phi(z)} e^{ \pm \phi\left(z^{\prime}\right)}=e^{ \pm \phi\left(z^{\prime}\right)} e^{\mp \phi\left(z^{\prime}\right)} e^{\phi(z)} e^{ \pm \phi\left(z^{\prime}\right)}=-e^{ \pm \phi\left(z^{\prime}\right)} e^{\phi(z)}
$$

provided for $\left|z^{\prime}\right|=|z|$ we have

$$
\left[\phi\left(z^{\prime}\right), \phi(z)\right]= \pm i \pi \quad \text { which implies } \quad \phi(z) \phi(0) \sim \ln z
$$

Now, if we are given more than one pair of fermions, they won't naturally anticommute because $\left[\phi_{i}, \phi_{j}\right]=0$. This is corrected by the introduction of cocycles $[6]$ :

- Order all bosons of the theory: $\phi_{i}$ where $i=1, \ldots, N$;

- Then multiply each exponential by a factor $(-)^{N_{1}+\ldots+N_{i-1}}$, where $N_{i}$ is the fermion number operator:

$$
N_{i}=-\oint \frac{\mathrm{d} z}{2 \pi i} \bar{\psi}_{i} \psi_{i}=\oint \frac{\mathrm{d} z}{2 \pi i} \partial \phi_{i}
$$

For example, if we consider two pairs of fermions, the bosonization becomes

$$
\psi_{1}=e^{\phi_{1}}, \quad \bar{\psi}_{1}=e^{-\phi_{1}}
$$

with

$$
\psi_{2}=e^{\phi_{2}}(-)^{N_{1}}, \quad \bar{\psi}_{2}=e^{-\phi_{2}}(-)^{N_{1}}
$$

where now $\psi_{1}$ and $\psi_{2}$ anticommute

$$
e^{\phi_{1}} e^{\phi_{2}}(-)^{N_{1}}=e^{\phi_{2}} e^{\phi_{1}}(-)^{N_{1}}=e^{\phi_{2}}(-)^{N_{1}}(-)^{-N_{1}} e^{\phi_{1}}(-)^{N_{1}}=-e^{\phi_{2}}(-)^{N_{1}} e^{\phi_{1}}
$$

provided

$$
\left[N_{i}, e^{n \phi_{j}}\right]=n \delta_{i j} e^{n \phi_{j}} .
$$

Thus, for more than one pair of fermions, we need to introduce the cocycle phase factors:

$$
c_{i}=(-)^{N_{1}+\ldots+N_{i-1}}
$$

Consider the vector

$$
\partial \phi=\left(N_{1}, N_{2}, \ldots, N_{5}\right)
$$

\footnotetext{
${ }^{2}$ https://en.wikipedia.org/wiki/Baker-Campbell-Hausdorff_formula.
} 
then the cocycle factor can be written as

$$
c_{ \pm e_{i}}=\exp \left[ \pm i \pi\left\langle e_{i} M \partial \phi\right\rangle\right]
$$

where $e_{i}$ is 1 in the $i$ th component and zero elsewhere, \langle\rangle is a matrix inner product and $M$ is a lower triangular matrix with entries \pm 1 :

$$
M=\left(\begin{array}{cccccc}
0 & 0 & 0 & 0 & 0 & 0 \\
1 & 0 & 0 & 0 & 0 & 0 \\
1 & 1 & 0 & 0 & 0 & 0 \\
-1 & 1 & -1 & 0 & 0 & 0 \\
1 & 1 & 1 & 1 & 0 & 0 \\
-1 & -1 & -1 & -1 & -1 & 0
\end{array}\right)
$$

The signs of $M$ are arbitrary at this point, but they can be specified studying the charge conjugation matrix [6].

The cocycle factors of spin fields, $c_{\alpha}$ and $c_{\dot{\alpha}}$, are given the following expressions:

$$
c_{\alpha}=\exp [i \pi\langle\alpha M \partial \phi\rangle] \quad \text { and } \quad c_{\dot{\alpha}}=\exp [i \pi\langle\dot{\alpha} M \partial \phi\rangle]
$$

Gamma matrices. To motivate the construction of gamma matrices and show how cocycles work, let us consider the OPE between $\psi^{i}$ and $S^{\alpha}$. Using expressions (2.4) and (A.6) we have to compute the OPE of $e^{\phi_{i}(z)} c_{i}$ with $e^{\alpha \phi(\mathrm{w})} c_{\alpha}$. Notice that $c_{i}$ will pass through $e^{\alpha \phi}$ and due to Baker-Campbell-Hausdorff we obtain an extra phase:

$$
c_{i} e^{\alpha \phi}=e^{i \pi\left\langle e_{i} M \partial \phi\right\rangle} e^{\alpha \phi}=e^{i \pi\left\langle e_{i} M \alpha\right\rangle} e^{\alpha \phi} c_{i}
$$

so that our OPE becomes

$$
e^{\phi_{i}(z)} c_{i} e^{\alpha \phi(\mathrm{w})} c_{\alpha} \sim(z-\mathrm{w})^{\alpha \cdot e_{i}} e^{i \pi\left\langle e_{i} M \alpha\right\rangle} e^{\left(e_{i}+\alpha\right) \phi} c_{i+\alpha} .
$$

Notice that we obtain a branch-cut if $\alpha \cdot e_{i}=\alpha_{i}=-1 / 2$ which in turn implies that the sum $e_{i}+\alpha$ must be an anti-chiral index $\dot{\beta}$. Therefore given

$$
e^{\phi_{i}(z)} c_{i} e^{\alpha \phi(\mathrm{w})} c_{\alpha} \sim(z-\mathrm{w})^{-1 / 2} e^{i \pi\left\langle e_{i} M \alpha\right\rangle} e^{\dot{\beta} \phi} c_{\dot{\beta}},
$$

we see that it becomes natural to define the gamma matrices as

$$
\left(\Gamma^{j}\right)_{\dot{\alpha}}^{\beta}=\sqrt{2} \delta\left(e_{j}+\beta-\dot{\alpha}\right) e^{i \pi\left\langle e_{j} M \dot{\alpha}\right\rangle}
$$

and

$$
\left(\Gamma^{j}\right)_{\alpha}^{\dot{\beta}}=\sqrt{2} \delta\left(e_{j}+\dot{\beta}-\alpha\right) e^{i \pi\left\langle e_{j} M \alpha\right\rangle}
$$

giving us the final result:

$$
\psi^{i}(z) S^{\alpha}(\mathrm{w}) \sim \frac{1}{\sqrt{2}} \frac{\Gamma_{\dot{\beta}}^{i \alpha} S^{\dot{\beta}}(\mathrm{w})}{(z-\mathrm{w})^{1 / 2}} .
$$

The explicit representation is written in terms of the Pauli-matrices via

$$
\Gamma^{ \pm e_{j}}=( \pm i)^{j-1} \sqrt{2}\left(\sigma^{3} \otimes\right)^{j-1} \sigma^{\mp}(\otimes 1)^{5-j}
$$


and one can convert between $u(5)$ and covariant so(10) using

$$
\Gamma^{2 j-1}=\frac{1}{\sqrt{2}}\left(\Gamma^{e_{j}}+\Gamma^{-e_{j}}\right)
$$

and

$$
\Gamma^{2 j}=\frac{i}{\sqrt{2}}\left(\Gamma^{e_{j}}-\Gamma^{-e_{j}}\right)
$$

Notice that in our construction, the notation $\gamma^{\mu}$ is reserved for the symmetric gamma matrices:

$$
\begin{aligned}
\gamma_{\alpha \beta}^{\mu} & =\Gamma_{\alpha}^{\mu \dot{\beta}} C_{\dot{\beta} \beta} \\
\gamma^{\mu \alpha \beta} & =\Gamma_{\dot{\beta}}^{\mu \alpha} C^{\dot{\beta} \beta}
\end{aligned}
$$

as it is common in the literature. In above equations, $C$ denotes the charge conjugation matrix which is the next topic in our discussion.

Charge conjugation matrix. We define $C$ as

$$
C^{\beta \dot{\beta}}=\delta(\beta+\dot{\beta}) e^{i \pi \beta M \dot{\beta}}
$$

and

$$
C^{\dot{\beta} \beta}=-\delta(\dot{\beta}+\beta) e^{i \pi \dot{\beta} M \beta}
$$

and with these convetions we have $C^{\beta \dot{\beta}}=C^{\dot{\beta} \beta}$. These expressions can be motivated by studying the OPE of $S^{\alpha}$ and $S^{\dot{\beta}}$.

It is also common to use only undotted indices when describing spinors in 10d. Charge matrices act as metrics on the spinor space and can remove all dotted indices. For us all spinors are defined with upper indices and then anti-chiral ones are written as

$$
S_{\beta}=C_{\beta \dot{\beta}} S^{\dot{\beta}}
$$

This notation is used together with the symmetric gamma representation.

Open Access. This article is distributed under the terms of the Creative Commons Attribution License (CC-BY 4.0), which permits any use, distribution and reproduction in any medium, provided the original author(s) and source are credited.

\section{References}

[1] L. Mason and D. Skinner, Ambitwistor strings and the scattering equations, JHEP 07 (2014) 048 [arXiv: 1311.2564] [INSPIRE].

[2] F. Cachazo, S. He and E.Y. Yuan, Scattering of Massless Particles in Arbitrary Dimensions, Phys. Rev. Lett. 113 (2014) 171601 [arXiv:1307.2199] [INSPIRE].

[3] N. Berkovits and M. Lize, Field theory actions for ambitwistor string and superstring, JHEP 09 (2018) 097 [arXiv: 1807.07661] [INSPIRE]. 
[4] J. Fang and C. Fronsdal, Massless Fields with Half Integral Spin, Phys. Rev. D 18 (1978) 3630 [INSPIRE].

[5] D. Friedan, E.J. Martinec and S.H. Shenker, Conformal Invariance, Supersymmetry and String Theory, Nucl. Phys. B 271 (1986) 93 [inSPIRE].

[6] V.A. Kostelecky, O. Lechtenfeld, W. Lerche, S. Samuel and S. Watamura, Conformal Techniques, Bosonization and Tree Level String Amplitudes, Nucl. Phys. B 288 (1987) 173 [INSPIRE].

[7] I.G. Koh, W. Troost and A. Van Proeyen, Covariant Higher Spin Vertex Operators in the Ramond Sector, Nucl. Phys. B 292 (1987) 201 [INSPIRE]. 